\title{
Wild-Type Cone Photoreceptors Persist Despite Neighboring Mutant Cone Degeneration
}

\author{
Alaron Lewis, ${ }^{1}$ Philip Williams, ${ }^{2}$ Owen Lawrence, ${ }^{1}$ Rachel 0. L. Wong, ${ }^{2}$ and Susan E. Brockerhoff ${ }^{1}$ \\ Departments of ${ }^{1}$ Biochemistry and ${ }^{2}$ Biological Structure, University of Washington, Seattle, Washington 98195
}

In many retinal diseases, the malfunction that results in photoreceptor loss occurs only in either rods or cones, but degeneration can progress from the affected cell type to its healthy neighbors. Specifically, in human and mouse models of Retinitis Pigmentosa the loss of rods results in the death of neighboring healthy cones. Significantly less is known about cone-initiated degenerations and their affect on neighboring cells. Sometimes rods remain normal after cone death, whereas other patients experience a loss of scotopic vision over time. The affect of cone death on neighboring cones is unknown. The zebrafish is a cone-rich animal model in which the potential for dying cones to kill neighboring healthy cones can be evaluated. We previously reported that the zebrafish cone phosphodiesterase mutant $\left(p d e 6 c^{\mathrm{w} 59}\right.$ ) displays a rapid death of cones soon after their formation and a subsequent loss of rods in the central retina. In this study we examine morphological changes associated with cone death in vivo in $p d e 6 c^{\mathrm{w59}}$ fish. We then use blastulae transplantations to create chimeric fish with a photoreceptor layer of mixed wild-type (WT) and $p d e 6 c^{\mathrm{w} 59}$ cones. We find that the death of inoperative cones does not cause neighboring WT cone loss. The survival of WT cones is independent of transplant size and location within the retina. Furthermore, transplanted WT cones persist at least several weeks after the initial death of dysfunctional mutant cones. Our results suggest a potential for the therapeutic transplantation of healthy cones into an environment of damaged cones.

\section{Introduction}

Rods and cones are the primary light sensing cells of the visual system and form a mixed layer of cells in the outer retina. Cones are responsible for daytime and color vision whereas rods respond in low light levels. The phototransduction cascades of cones and rods are mechanistically analogous, but genetically distinct. Thus, mutations that abolish phototransduction in one cell type do not affect phototransduction in the other. However, in Retinitis Pigmentosa (RP), a rod-based disease, the death of inoperative rods causes the death of functional neighboring cones (Hartong et al., 2006). In patients, this secondary death manifests as tunnel vision and eventually blindness. The spread of death from damaged cells to their healthy neighbors is known as the bystander effect (Ripps, 2002). Understanding and controlling the bystander effect is one of the major challenges in the treatment of retinal degeneration.

Retinal diseases are particularly complex due to the variability of the bystander effect between different types of photoreceptors. Studies in humans and mouse models have shown that rod degeneration frequently results in the death of neighboring cones, although the extent of cone degeneration can vary between patients (Ripps, 2002; Delyfer et al., 2004; Hartong et al., 2006).

Received 0ct. 8, 2009; revised; accepted Nov. 12, 2009.

Funding was provided by the following grants from the National Institutes of Health: R01EY018814 (S.E.B.), EY14358 (R.0.L.), T32EY007031, 5F32EY019210 (A.A.L.) and the UW Vision Core facility Grant P30EY01733. We thank Hillary Kemp in the Moens laboratory for assistance with transplant technique and members of the Kimelman laboratory for helpful suggestions and transplant equipment.

Correspondence should be addressed to Susan E. Brockerhoff, University of Washington, Department of Biochemistry, Box 357350, Seattle, WA 98195. E-mail: sbrocker@u.washington.edu.

D0I:10.1523/JNEUROSCI.5019-09.2010

Copyright $\odot 2010$ the authors $\quad 0270-6474 / 10 / 300382-08 \$ 15.00 / 0$
Mosaic mouse models have further shown that degenerating rods will kill neighboring functional rods (Huang et al., 1993; Kedzierski et al., 1998). Thus, rod degeneration leads to the secondary degeneration of neighboring healthy rod and cone photoreceptors. In contrast, the spread of death from dying cones to their healthy photoreceptor neighbors is more unpredictable. In some cases of cone dystrophy, the rods are completely unaffected (Michaelides et al., 2004). In other cases, the degeneration of cones causes a decrease in scotopic vision presumably as a result of rod death (Hamel, 2007). It is unclear how these two types of dystrophy differ. Furthermore, due to a lack of cone-rich animal models, no studies have examined the bystander effect between cones. Since the maintenance of cones is extremely important for the treatment of retinal degeneration, a thorough understanding of the bystander effect between cones will influence therapeutic efforts of cone repopulation and transplantation.

Recently, we identified a zebrafish mutant deficient in cone phosphodiesterase $\left(p d e 6 c^{w 59}\right)$. In this mutant, cones rapidly degenerate after their formation and there is a subsequent loss of rods in the cone-dense central retina (Stearns et al., 2007). We exploited this phenotype, and the advantages of the zebrafish system, to conduct an in vivo study of cone-cone interaction in a degeneration paradigm. Zebrafish larvae have a cone-dominated retina and can be maintained in a translucent state to allow visualization of cones in the intact animal. Furthermore, chimeric animals containing mixed wild-type (WT) and mutant cone populations can be generated and cones can then be monitored in live animals to evaluate whether they degenerate. Using these techniques we show that there is not a cone-cone bystander effect even in areas of the retina where we previously detected rod death. This is the first evidence that large scale cone degeneration 
can occur without causing the secondary death of neighboring healthy cones that reside within the degenerating environment.

\section{Materials and Methods}

Plasmid production. To express the membrane targeted version of enhanced cyan fluorescent protein (MCFP) specifically in cones, we subcloned 3.2 Kb of the cone $\alpha$ transducin promoter (T $\alpha C P$ ) (Kennedy et al., 2007) and the MCFP (Morris et al., 2005) into a modified version of the tol 2 vector (Taylor et al., 2005). We generated T $\alpha C P$ driven Td-tomato and MCherry constructs using the Gateway-based tol2kit (Kwan et al., 2007). The resulting constructs were injected into WT zebrafish larvae together with tol2 transposase mRNA (Taylor et al., 2005) and germline carriers were identified in the $\mathrm{F} 1$ generation.

Zebrafish care and maintenance, and strains. Adult fish and larvae were maintained as described previously (Westerfield, 1995). Unless otherwise noted, all zebrafish strains are homozygous for the roy orbison (roy) mutation (Ren et al., 2002). Fish used in live imaging experiments were maintained in embryo media containing $0.003 \%$ 1-phenyl 2-thiourea (ptu) starting at $10 \mathrm{~h}$ postfertilization (hpf). The $p d e 6 c^{w 59}$ mutant was isolated in a screen of mutagenized zebrafish using the optokinetic response (OKR) behavioral assay as described previously (Stearns et al., 2007). To visualize dying cone photoreceptors we crossed the $p d e 6 c^{w 59}$ mutant strain to the stable transgenic fish lines, $\operatorname{Tg}(T \alpha C P: G F P)$ (Kennedy et al., 2007) or $\operatorname{Tg}(\operatorname{T\alpha CP:MCFP)}$ made in this study.

Live imaging. For time lapse imaging, $5 \mathrm{~d}$ postfertilization (dpf) larvae were transferred to a Petri dish (Falcon \# 35-3037) containing 0.5\% low melting point agarose dissolved in embryo medium containing $0.003 \%$ 1-phenyl 2-thiourea (ptu) and $0.02 \%(\mathrm{w} / \mathrm{v})$ Tricaine. During imaging the dish was placed in a custom made ITO glass heating system and maintained at $28^{\circ} \mathrm{C}$ using a temperature controller (TC2 bip, Cell Micro Controls). Time-lapse images were taken on a custom-built two-photon microscope at $890 \mathrm{~nm}$, using a $60 \times$ [numerical aperture (NA) 1.1] long working distance water objective. Image analysis was performed using MetaMorph (Universal Imaging) software. Adobe Photoshop CS was used to process the images further.

Blastulae transplants: production and counting. Blastulae transplants were performed as previously described (Carmany-Rampey and Moens, 2006). Mutant donors were identified by optokinetic response (OKR) analysis at $4 \mathrm{dpf}$ (Brockerhoff, 2006). Mutant hosts were identified by genotyping after final imaging (see below). Images of the transplants were taken using a custom-built two-photon microscope at $890 \mathrm{~nm}$, with a $20 \times$ (Olympus NA 1.0) long working distance water objective. Image analysis was performed using MetaMorph and Amira (NVIDIA) software. Amira was used to align initial and final images, and the cells were counted in each image stack. Each cell was marked in the three-dimensional reconstruction of the image stacks to ensure that all cells were counted only once. These counts were obtained independently for each time point. Adobe Photoshop CS was used to adjust brightness and contrast.

Pde6c genotyping. Genotyping of larvae were performed after the completion of each experiment. DNA preparation was done as described previously (Truett et al., 2000). Fish were genotyped by sequencing or by PCR using the following primers to create a restriction site for BsaX1 in only the mutant locus (forward: ttg gcc tctggaata ctg gct ctc, reverse: gtttgaccagaacccggaag). Products were cut with BsaX1 and genotyped by restriction profile.

Whole-mount TUNEL and 21 dpf cryosections. Fish were killed in tricaine, the heads were removed and put into $4 \%$ paraformaldehyde in $1 \times \mathrm{PBS}$ for $1 \mathrm{~h}$, and bodies were processed for genotyping. For TUNEL staining, heads were washed twice in $1 \times$ PBS and the eyes were removed. Eyes were washed twice in PBSTT (PBS $+1 \%$ Triton X100 + 1\% Tween) and treated with Proteinase $\mathrm{K}(5 \mathrm{ng} / \mu \mathrm{l})$ for $10 \mathrm{~min}$. The eyes were washed once in PBSTT, refixed in 4\% PFA (in PBSTT) for $20 \mathrm{~min}$ at room temperature, and washed three times in PBSTT and twice in PBS. TUNEL was done using the ApopTag Red In Situ Apoptosis detection Kit (Millipore Bioscience Research Reagents) according to manufacturer's instructions with the following changes. Equilibration buffer was applied for $30 \mathrm{~min}$. Eyes were incubated in blocking buffer for $2 \mathrm{~h}$ at $4^{\circ} \mathrm{C}$ before application of anti-digoxigenin conjugate, and they remained in conjugate at $4^{\circ} \mathrm{C}$ in a humidified chamber overnight before washing and im- aging. Cryosections of $21 \mathrm{dpf}$ fish were obtained as described previously (Stearns et al., 2007). Laser scanning confocal microscopy was performed using an Olympus FV1000, 60× (NA 1.34) oil-immersion lens. Image analysis was performed using MetaMorph. Adobe Photoshop CS was used to adjust brightness and contrast.

\section{Results}

\section{The morphology of cone death in pde6 $c^{\text {w59 }}$}

To identify photoreceptors undergoing cell death in vivo, we determined the morphological changes of cones in the $p d e 6 c^{\mathrm{w} 59}$ background. This was performed using a MCFP under the control of the cone-specific promoter (T $\alpha C P)$ (Kennedy et al., 2007). The $T \alpha C P$ promoter drives expression in all cone types starting at 3 dpf (Kennedy et al., 2007). MCFP labels all membranes throughout the cone and accumulates in the outer segments, thus enabling both the counting of cells in a crowded environment and a detailed examination of cone morphology during degeneration (Fig. 1).

To visualize the changes in individual cones, embryos at the one cell stage were injected with a plasmid containing $T \alpha C P$ : $M C F P$. This injection results in transient mosaic expression of MCFP throughout the cone population. At $3 \mathrm{dpf}$, when MCFP is initially expressed, both mutant and wild-type cones show an immature morphology including a small outer segment and a well formed pedicle (data not shown). Over the next $24 \mathrm{~h}$ wildtype cones enlarge their outer segments (Fig. 1), and by day 4 the fish display a robust optokinetic response (Brockerhoff, 2006). In the $p d e 6 c^{\text {w59 }}$ mutant, the outer segments elongate poorly and by $4 \mathrm{dpf}$, many of the cones in the center of the retina begin to undergo cell death (Fig. 1).

The process of photoreceptor death takes several hours to complete. At $4 \mathrm{dpf}$, $p d e 6 c^{\text {w59 }}$ cones still have relatively short outer segments while the pedicle is broad and contains multiple small processes. The first noticeable indication of cone death is the retraction of these processes. This is followed by a complete withdrawal of the pedicle from the outer plexiform layer and shrinkage of the outer segment, reducing the bright MCFP signal (Fig. 1). Subsequently, the cell begins to round-up and lose all traces of the outer segment. This process, from filopodial retraction to the formation of a rounded apoptotic body, takes 6-8 h to occur. Eventually, the apoptotic body is transported through the outer plexiform layer and removed from the retina (data not shown).

In contrast to the dramatic morphological changes seen in pde $6 c^{w 59}$ mutant cones, wild-type cones show few changes over this same time frame (Fig. 1).

\section{The death of cones is biphasic and the majority of cones die in $1 \mathrm{~d}$}

Recently, Punzo et. al. (2009) reported that in several mouse models of RP the degeneration of the rod population occurs as a two-step process. They found that all mutants had an initial rapid phase of death in which the majority of rods died, followed by a much slower death of the remaining rod population. Given these findings, we determined whether the death of the cone population in our $p d e^{w 59}$ zebrafish mutant was also biphasic.

To count the number of cells in the retina that had died at each time point it was necessary to visualize the entire cone population. Thus, we produced a stable transgenic line of T $\alpha C P: M C F P$. Similar to transiently injected animals, MCFP expression marks the outer membrane, outer segments and pedicles of cones starting at $3 \mathrm{dpf}$. However, all the cones are labeled in these animals. Cone outer segments in $T g(T \alpha C P: M C F P)$ animals were bright and neighboring outer segments could be distinguished allowing 
us to quantify the number of cones in each region of the eye. Wild-type $\operatorname{Tg}(T \alpha C P$ : $M C F P$ ) have a normal optokinetic response, indicating that this transgene does not affect vision.

To examine timing of cone death throughout the retina, we crossed the $\mathrm{Tg}$ (T $\alpha C P: M C F P)$ into the $p d e 6 c^{\mathrm{w} 59} \mathrm{mu}-$ tant background. These fish were killed and fixed and their eyes removed and placed, lens down, onto a microscope slide. Since our morphological analysis of individual cones showed that the outer segment disappeared as cells were undergoing cell death, we used the presence of an outer segment to count cones that had not yet died. In teleosts, new cones are generated in the peripheral margins of the retina throughout the life of the fish and thus, $p d e 6 c^{\mathrm{w} 59}$ fish always contain immature cones in the retinal periphery. The inversion of the eye allowed visualization of the central retina where the photoreceptors are the most mature. We started our analysis at $4 \mathrm{dpf}$ since this is when cones begin to degenerate in this mutant.

In $p d e 6 c^{\mathrm{w} 59}$ retinas, there is a rapid decrease in MCFP fluorescence between 4 and $7 \mathrm{dpf}$ (Fig. 2B). Similar to studies of rods in the $r d 1$ mouse, we found that the decrease in the number of fluorescent cone outer segments occurred in two phases. In the initial rapid stage of death $60 \pm 11 \%$ of the cones in the central retina lost their outer segment fluorescence within a $24 \mathrm{~h}$ period between 4 and $5 \mathrm{dpf}$ (Fig. 2C). The remaining cells often appeared unhealthy having small outer segments and a flattened morphology (Fig. 2B). These residual dystrophic cells disappeared more slowly and an additional $19 \pm 9 \%$ decrease in cell number occurred over the next $2 \mathrm{~d}$ (Fig. 2C). Thus, by $7 \mathrm{dpf}, 70-88 \%$ of the cones in the center of the mutant retina have died. In contrast, wild-type eyes maintain a constant density of cones throughout this period (Fig. 2A,C).

To confirm that cells were dying by apoptosis and that remaining cells in the central retina with bright outer segments had not undergone apoptosis, we used the TUNEL assay. The presence of TUNEL staining indicates the cleavage of DNA into a 200bp ladder, which is a hallmark of late stage apoptotic cells. TUNEL staining was performed on dissected whole mount retinas from both $p d e 6 c^{\text {w59 }}$ and wild-type fish containing $\operatorname{Tg}(\mathrm{T} \alpha \mathrm{CP}: \mathrm{MCFP})$ (Fig. $3 \mathrm{~A}, \mathrm{~B})$. At 4 $\mathrm{dpf}$, the $p d e 6 c^{\mathrm{w} 59}$ retina shows only a minor increase in the levels of TUNEL staining compared to WT. By $5 \mathrm{dpf}$, the density of TUNEL-positive cells in the mutant retinas is fourfold greater than either wild-type or heterozygous siblings, and remains high through 6 and $7 \mathrm{dpf}$ (Fig. 3C). In images of individual cells such as those shown in Figure 1, several apoptotic bodies persisted in the outer nuclear layer (ONL) for the duration of our $8 \mathrm{~h}$ time tion. Scale bar, $10 \mu \mathrm{m}$.
WT 5dpf
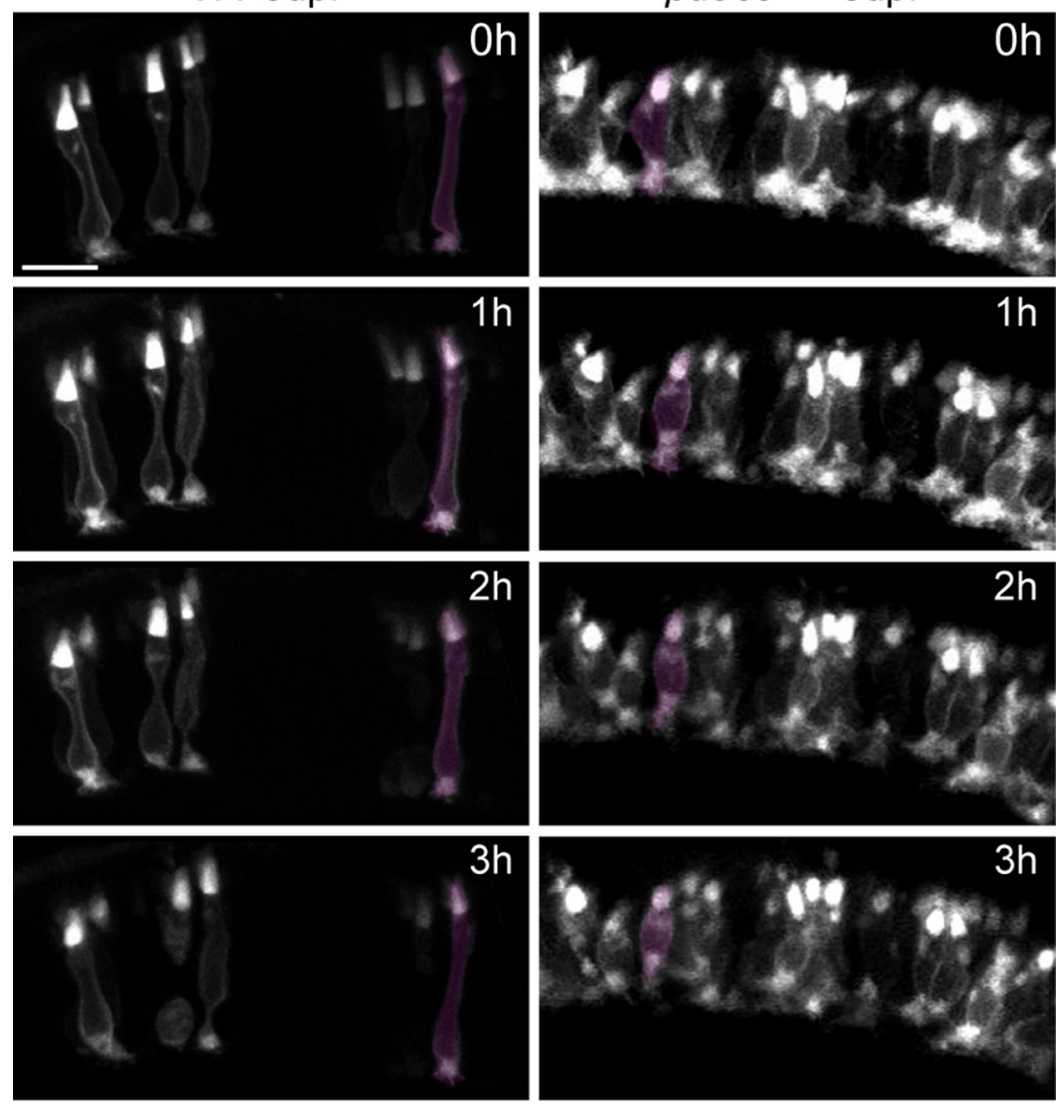

$4 \mathrm{~h}$
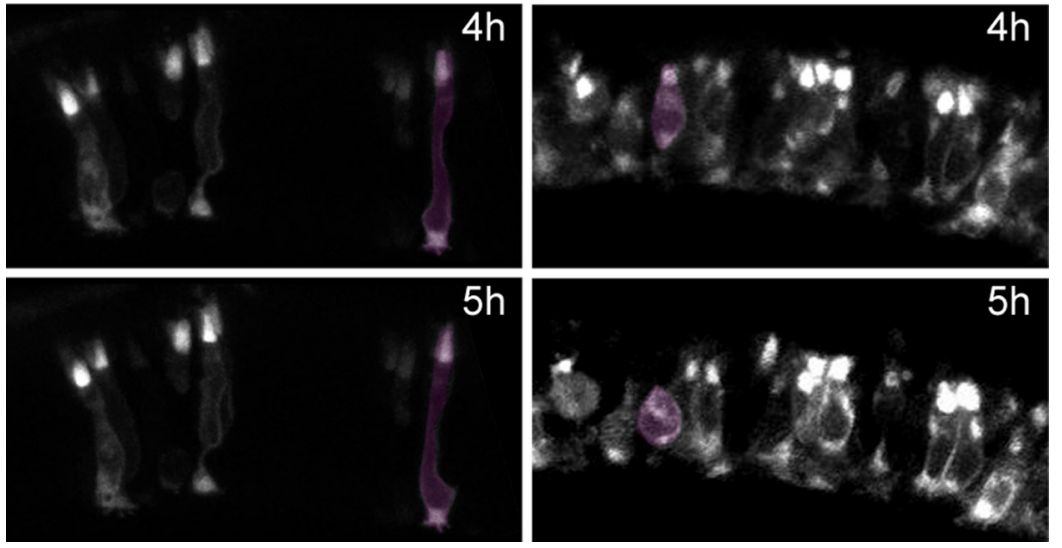

$5 h$

Figure 1. Morphological changes in individual cones undergoing death in the $p d e 6 c^{\mathrm{w} 59}$ mutant. Wild-type and mutant embryos were injected with $T \alpha C P$ :MCFP at the one-cell stage. At $4 \mathrm{dpf}$, mosaic patches of MCFP-positive cones were imaged once an hour for $7 \mathrm{~h}$. One cell in each genotype was pseudocolored throughout the time course of the recording to facilitate its identifica-

courses (data not shown). Thus, the further increase in TUNEL-positive cell density in day 6 and 7 images may be due to the persistence of a small percentage of the apoptotic bodies from earlier phases of cell death.

To confirm that MCFP-positive cells in the back of the eye had not yet undergone apoptosis, we compared MCFP expression and TUNEL staining in the mutant eye. Although the density of TUNELpositive cells in $p d e 6 c^{\text {w59 }}$ fish was significantly above wild type at all time points, most cells showing continued MCFP staining in their outer segments did not have TUNEL staining (Fig. 3B). This confirms that these cells have not yet undergone apoptosis and do represent a second phase of more slowly progressing cone death in the pde $6 c^{\mathrm{w} 59}$ mutant. 


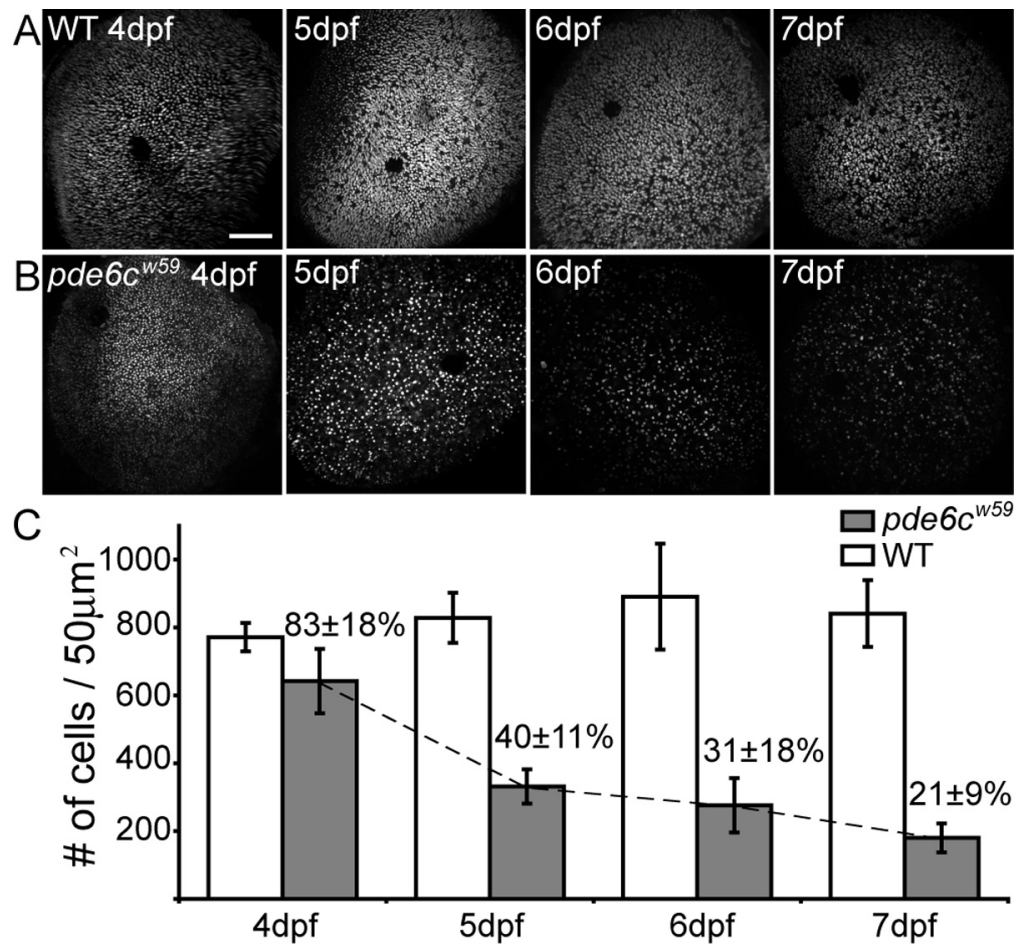

Figure 2. Death of the cone population occurs in two stages in $p d e 6 c^{\text {w59 }}$. $A, B$, Eyes from wild-type $(\boldsymbol{A})$ and $p \operatorname{de} 6 c^{\mathrm{w} 59}(\boldsymbol{B})$ $\operatorname{Tg}(T \alpha C P: M C F P)$ fish were removed at the indicated ages and inverted onto a slide to allow imaging of the cones in the central retina at the back of the eye. Scale bar, $50 \mu \mathrm{m}$. C, Cell counts show a rapid decline in cone number in pde6c ${ }^{\text {w59 }}$ between 4 and $5 \mathrm{dpf}$ followed by a slow loss over the next several days. Percentages are $p d e 6 c^{\text {w59 }}$ over wild-type cell counts at each time point, and error bars are the SEM.

\section{pde $6 c^{\text {w59 }}$ cone death is cell autonomous}

Studies of mosaics using the $r d 1$ and wild-type rods showed that the death of $r d 1$ rods was cell autonomous and occurred even in a wild-type environment (Huang et al., 1993; Kedzierski et al., 1998). We were interested to see whether a local wild-type environment could prevent $p d e 6 c^{\mathrm{w} 59}$ mutant cone death or whether the death of cells containing the $p d e 6 c^{\text {w59 }}$ mutation would also be autonomous, similar to $r d 1$ rods. To examine $p d e 6 c^{\text {w59 }}$ cones in a wild-type environment, we transplanted mutant cells into wildtype host blastulas. To distinguish donor from host cones, we used a two-color system in which the $T \alpha C P$ promoter drove the expression of different fluorophores in the host and donor cones. We evaluated several fluorophores to determine which would be most useful for our experiments. We had previously constructed the transgenic line expressing GFP in cones, $\operatorname{Tg}(T \alpha C P: G F P)$ (Kennedy et al., 2007), and we used the tol 2 system to generate fish strains containing td-Tomato or MCherry as well. We found that both red fluorophores were too dim at $3 \mathrm{dpf}$ to accurately visualize transplant number and position. However, the $\mathrm{Tg}(\mathrm{T} \alpha \mathrm{CP}: G F P)$ is easily detected and was therefore used to mark transplanted cones. Unlike MCFP, GFP expression in this transgenic line is cytosolic and does not accumulate in photoreceptor outer segments. Thus, the loss of outer segment fluorescence could not be used as a measure of cell death and instead GFPpositive cells were scored as either present or absent. As a control, we analyzed $p d e 6 c^{w 59} \mathrm{Tg}(\mathrm{T} \alpha C P: G F P)$ cones transplanted into the $p d e 6 c^{w 59} \operatorname{Tg}(T \alpha C P: M C F P)$ fish and found that the majority of these cells were lost over time (Fig. 4A). This indicates that cone death can also be scored using GFP as a marker.

To generate chimeric animals, cells were removed from a donor embryo containing $\operatorname{Tg}(T \alpha C P: G F P)$ at the blastulae stage, $\sim 6 \mathrm{~h}$ postfertilization, and injected into a host embryo containing $\mathrm{Tg}(\mathrm{T} \alpha \mathrm{CP}$ : $M C F P)$. Thus, the host cones were visualized by MCFP expression and the donor cones were visualized by GFP expression. Initial images of the eye were acquired at 3 $\mathrm{dpf}$ to assess the number and location of the transplanted cells within the host retina (Fig. $4 A-D$ ). Fish were then allowed to mature for several days and between 6 and $9 \mathrm{dpf}$, the eye was again imaged in the intact animal (Fig. $4 A-D$ ). Since, as the fish age the lens can distort the image of cells in the center of the retina, we also removed the eye and inverted it for imaging at the final time point (supplemental Fig. 1, available at www.jneurosci.org as supplemental material). Transplanted cells were marked for quantification as shown in Figure 4. Small variations in cell location prevented a cell by cell identification between the two ages. However, separate patches of cells remained discrete and could still be differentiated from each other even after several days of eye growth (Fig. 4A-D). In some instances, images of older fish contained patches of transplant in the periphery of the eye that were not seen in the initial image, presumably due to continued generation of cones in the peripheral marginal zone (data not shown). In all of these cases the new patch could be differentiated from patches seen in the $3 \mathrm{dpf}$ images, and the counts from these new patches were not included.

In both mutant and wild-type hosts, at least $50 \%$ of mutant donor cells died by $6 \mathrm{dpf}$ (Fig. $4 A, B$ ). As was seen in the $p d e 6 c^{\mathrm{w} 59}$ mutant alone, the $p d e 6 c^{\text {w59 }}$ donor cells underwent a major round of apoptosis between 4 and $5 \mathrm{dpf}$. Figure 4 shows a representative image of $p d e 6 c^{\mathrm{w} 59}$ donor cells in a $p d e 6 c^{\mathrm{w} 59}$ host (Fig. $4 A$ ) or in a WT host (Fig. $4 B$ ). Multiple fish were counted for each genotype and the average percentage of cells remaining after $6 \mathrm{dpf}$ is shown in Figure $4 E$. There was no statistically significant difference between the number of $p d e 6 c^{\mathrm{w} 59}$ donor cells remaining in WT versus $p d e 6 c^{\text {w59 }}$ host fish (Fig. $4 E$ ). The level of $p d e 6 c^{\text {w59 }}$ donor cell death was also independent of the size of the transplant (supplemental Fig. 2, available at www.jneurosci.org as supplemental material). Thus, $p d e 6 c^{\mathrm{w} 59}$ cone death is cell autonomous.

\section{pde6 $c^{\mathrm{w} 59}$ cones do not cause a bystander effect in WT cones}

We next examined the potential for the death of $p d e 6 c^{\text {w59 }}$ mutant cones to affect neighboring wild-type cones. To do this we used blastula transplantation to produce chimeric retinas containing a variable number of WT cells in the mutant host retina. As a control, we transplanted WT cells into a WT host background. All of the hosts expressed $T g(T \alpha C P: M C F P)$ and the WT donors expressed $\operatorname{Tg}(\mathrm{T} \alpha C P: G F P)$. Images were taken at $3 \mathrm{dpf}$ to document the transplant size and location within the eye, and final images were taken between 6 and 9 dpf. Cells were counted and compared at each time point.

There was no significant difference between the viability of cells transplanted into WT or mutant hosts. As expected, wildtype cones transplanted into wild-type hosts showed little to no death in our experiments. However, surprisingly, when we 


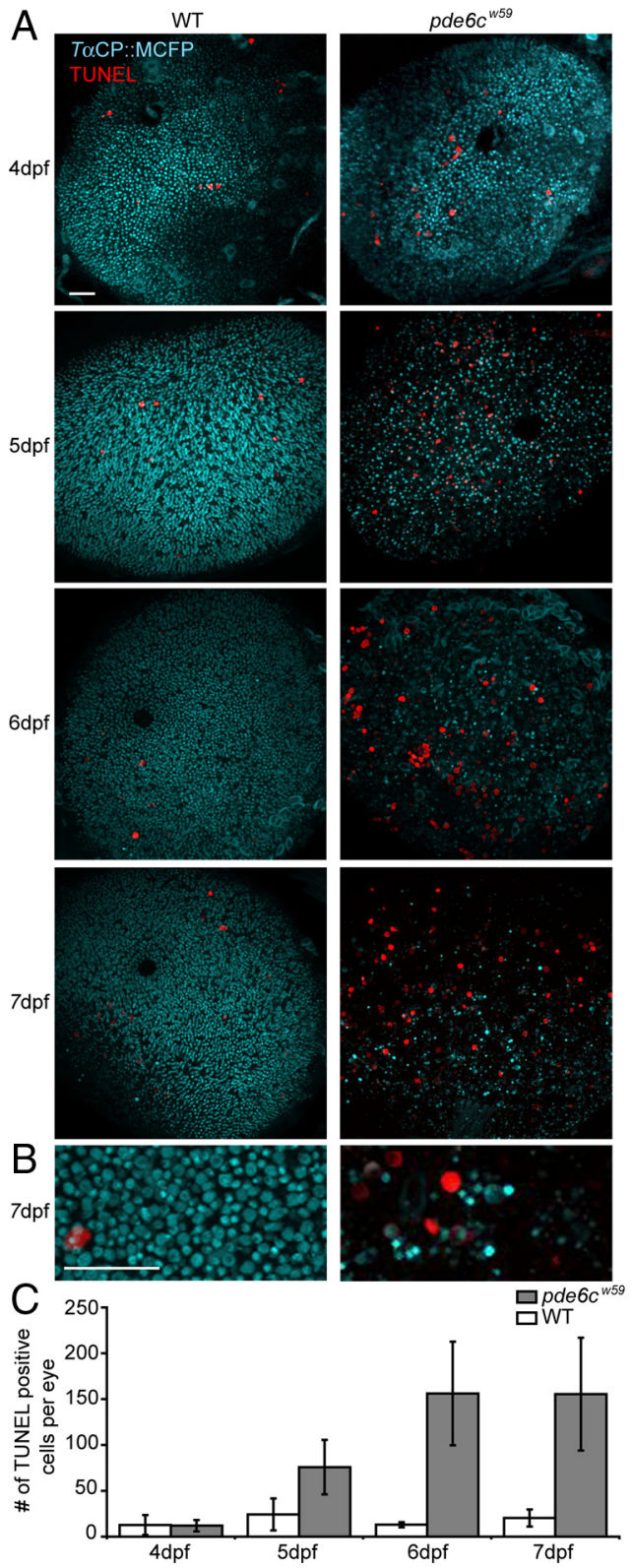

Figure 3. Mutant eyes have an increased density of TUNEL-positive cells. $A$, Eyes from wildtype and $p d e 6{ }^{\mathrm{w} 59} \mathrm{Tg}(\mathrm{T} \alpha$ (P:MCFP) fish were removed at the indicated ages and immunolabeled with TUNEL to show late stage apoptotic cells. Scale bar, $50 \mu \mathrm{m}$. $\boldsymbol{B}$, Higher magnification images show that most MCFP-positive cells are not TUNEL positive. Scale bar is $50 \mu \mathrm{m}$. C, The number of TUNEL-positive cells per eye is increased in mutant eyes as compared to wild-type siblings. Error bars are the SEM.

counted WT cells transplanted into pde $6 c^{\mathrm{w} 59}$ hosts, we found no significant loss of the WT cells. Representative images of WT donor cells transplanted into WT hosts (4C) or pde6 $c^{\text {w59 }}$ hosts (4D) are shown. Final images show that $90-100 \%$ of WT donor cells remained regardless of the genotype of the host backgrounds (Fig. $4 E$ ). To verify that mutant host cones were in fact dying in our experiments, MCFP-positive outer segments were examined in host retinas, demonstrating that a majority of the host cones had undergone apoptosis (Fig. 4D).

To determine whether there were morphological changes occurring in surviving WT cones, we performed a transplant of WT $\mathrm{Tg}(\mathrm{T} \alpha \mathrm{CP}: \mathrm{MCFP})$ donors into nonfluorescent $p d e 6 c^{\mathrm{w5} 9}$ hosts. The membrane localization of MCFP and the lack of host fluorescence allowed detailed visualization of WT cell morphology in the mutant background. At $3 \mathrm{dpf}$, transplants appear healthy and show no morphological abnormalities (Fig. $4 F, G$ ). At 5 dpf, WT cones in the periphery of the retina, where $p d e 6 c^{\mathrm{w} 59}$ cones persist, showed no morphological changes (Fig. 4F). However, in more central mutant retina, WT cones were often shorter and rounder showing a slightly flattened appearance (Fig. $4 G$ ). These cells are still present several days thereafter and continued to have outer segments and pedicles indicating that the cell shape changes are not due to an initiation of apoptosis. Instead, these morphological changes are probably due to the structural loss of neighboring cells and subsequent shrinkage of the ONL.

\section{WT viability was independent of size or position of the transplant}

Previous work with the $p d e 6 c^{\mathrm{w} 59}$ mutant showed that rods in the central retina die as the cones undergo apoptosis (Stearns et al., 2007). This effect was specific to central retina where the ratio of cones to rods is highest. We had proposed that the abundance of dying cones in this region was responsible for the initiation of rod death (Stearns et al., 2007). Although transplanted WT cones did not appear to die in the mutant environment, we asked whether survival depended on the location within the retina. Thus, we compared the survival rates of independent groups of transplants in various regions of the retina.

For these measurements, the retina was divided into three regions; the central retina, defined as the central area with half of the total radius of the eye; the periphery, defined as a ring at the outer edge of the eye with a width that is one fourth of the total radius of the eye; and the intermediate zone between the two. The images of excised and inverted eyes show primarily the central retina, and confirm that there is a uniform level of cone death over this entire region (Figs. 2, 3). We expected the peripheral zones of the retina to show limited death of cones due to continued proliferation within this region. Separation of the WT transplants into the three regions was performed using the $3 \mathrm{dpf}$ image and each region was independently compared to the final image. If an increased density of dying cones affected cones and rods similarly, then WT transplants should show reduced viability in the central region of the retina. However, there was no difference in the viability of WT cone transplants within the three regions of the eye (Fig. 5A). Transplanted cones survived in the central retina as well as in the periphery. Thus, even in areas of the retina where rods are dying, wild-type cones persist.

Additionally, we compared viability across transplant size, because rods are not abundant in the central retina of zebrafish larvae, and larger WT transplants might protect interior cells from exposure to the $p d e 6 c^{\mathrm{w} 59}$ environment. Transplants were examined for small groups of cells that were isolated from each other and these groups were counted individually and graphed according to transplant size (Fig. 5C). Our results indicate that smaller transplants show a slightly higher probability for cell loss, but this is independent of the genotype of the host suggesting that it is a property of the transplant procedure rather then a bystander effect. There were also several occasions where isolated WT cones persisted in the $p d e 6 c^{\mathrm{w} 59}$ host. Thus, even individual WT cones can survive in a cone degenerating environment (Fig. $5 B$ ). This suggests that, unlike rods, WT cones do not undergo a bystander effect as $p d e 6 c^{\text {w59 }}$ cones die.

\section{WT clones persist for at least several weeks in the} mutant environment

Our data indicates that wild-type transplants survive the initial death of cones in the mutant environment. However, the by- 


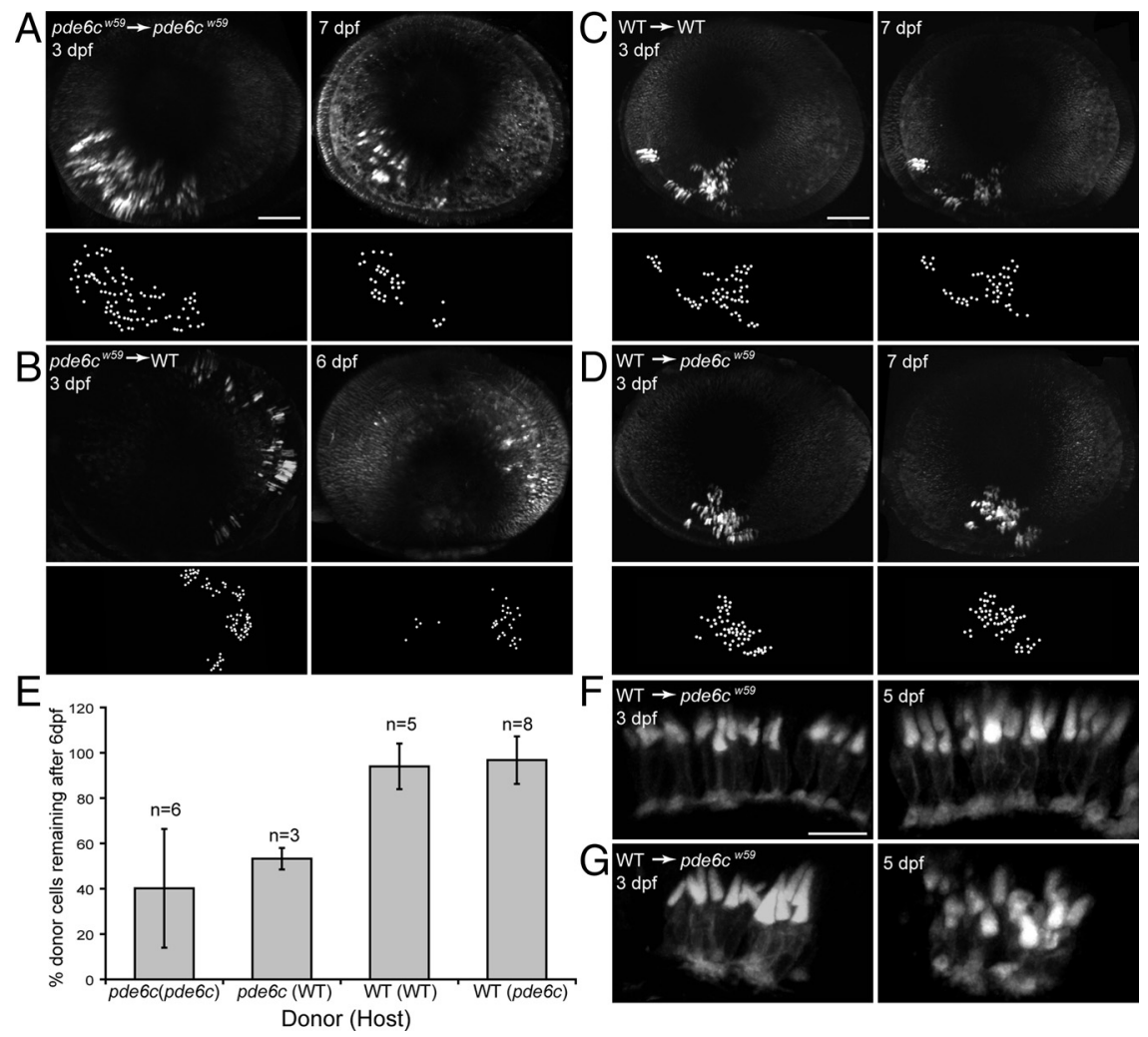

Figure 4. There is no cone to cone bystander effect in the $p d e 6 c{ }^{w 59}$ mutant. Blastulae stage transplants were performed to create chimeric fish containing a mixed photoreceptor layer. $\boldsymbol{A}-\boldsymbol{D}$, All cones are shown in gray scale. Donor cones express GFP (bright cytoplasmic fill), host cones express MCFP (outer segment fluorescence, visible in $7 \mathrm{dpf}$ images). Genotypes are listed as donor $\rightarrow$ host. Transplant size is determined at $3 \mathrm{dpf}$, and compared to images at $6-9 \mathrm{dpf}$. Scale bar, $50 \mu \mathrm{m}$. Counts below the images show a dot for each transplanted cone within the associated image above. $\boldsymbol{A}$, Mutant cells die in a mutant host. $\boldsymbol{B}$, Mutant cells die in a wild-type host. $\boldsymbol{C}$, Wild-type cells are viable in a wild-type host. $\boldsymbol{D}$, Wild-type cells are viable in a mutant host. $\boldsymbol{E}$, The viability of multiple transplants such as those in $A-D$ was graphed as the percentage of cells from the $3 \mathrm{dpf}$ counts that remained after $6 \mathrm{dpf}$. Error bars are the SEM, and $n=$ the number of fish. $\boldsymbol{F}, \mathbf{G}$, Higher magnification images of $T g(T \alpha C P: M C F P)$ donors in a pde6c ${ }^{w 59}$ host show some shortening of WT cones in the central retina. Host cones (mutant) are not fluorescent. Scale bar, $10 \mu \mathrm{m}$.

stander effect in the retina can often occur long after the primary degeneration. Thus, we examined older fish to ascertain whether the transplants survived over several weeks. Host fish transplanted with WT donors were imaged at $3 \mathrm{dpf}$ to confirm the presence of donor cones in the eye, and then grown to $21 \mathrm{dpf}$. Fish retinas were then cryosectioned and examined for GFP-positive donor cones. All hosts, both wild type $(n=4)$ and mutant $(n=$ 5), had GFP-positive cones, indicating that transplants survived in all cases. At this age, the $p d e 6 c^{\mathrm{w} 59}$ retina has almost no cones in the central retina (Fig. 6A) and only a small number in the peripheral marginal zone (data not shown). Additionally, there were not many rods in the central $p d e 6 c^{\mathrm{w} 59}$ retina, as indicated by the limited size of the ONL (Fig. 6A). Despite this dramatic absence of viable photoreceptors within the central retina of the pde $6 c^{\text {w59 }}$ mutant, the transplanted cones were present and had pedicles indicating that they were not undergoing apoptosis. However, transplanted WT cones in the mutant background were shorter than those in the WT hosts (Fig. 6B). This morphology is similar to what was observed at $5 \mathrm{dpf}$ (Fig. $4 G$ ) and is probably due to the collapse of the ONL. The presence of GFPpositive cones at $21 \mathrm{dpf}$ indicates that transplanted WT cones remain viable in a mutant environment devoid of endogenous photoreceptors even several weeks after degeneration has occurred.

\section{Discussion}

The bystander effect is a devastating aspect of retinal disease, leading to the degeneration of healthy functional photoreceptors. Although rod-initiated bystander effects are well documented in humans and mouse models, the ability of dying cones to influence healthy neighboring photoreceptors has not been carefully evaluated and is relevant to transplantation therapies. In this study, we exploit the experimental advantages of zebrafish such as optical clarity, cone dominance and ease of generating mosaic embryos to evaluate the influence of dying $p d e 6 c^{\mathrm{w} 59}$ cones on neighboring WT cones. Our study shows that cones can remain viable even as neighboring cones deteriorate.

In the $p d e 6 c^{\mathrm{w} 59}$ retina, cones die due to a null mutation in the cone-specific phosphodiesterase (Stearns et al., 2007). In this study, we examined the timing of this death in more detail. Similar to the death of rods in the $r d 1$ background, cone death occurs in two phases, a major phase between 4 and $5 \mathrm{dpf}$ during which time $60 \pm$ $11 \%$ of the cones die and a slower secondary phase in which the remaining cones eventually die. Using a membrane-targeted version of CFP we also visualized the detailed morphology of the dying cones. We found that filopodial protrusions retracted, the pedicle detached from the OPL and then the outer segment disappeared. The remaining rounded apoptotic cell body persisted for many hours before being cleared from the retina.

Previous studies showed that cone death was followed by a period of rod death in the central retina (Stearns et al., 2007). In this study we use blastulae stage transplantation to create chimeric fish that have a mosaic cone population in which wild-type and $p d e 6 c^{\text {w59 }}$ cones are adjacent to one another. Unlike native rods, which have degenerated by $8 \mathrm{dpf}$, wild-type cones remained viable long after the death of their $p d e 6 c^{\text {w59 }}$ neighbors. We investigated two possible differences between the native rods and our cone transplants that might account for this disparity. First, within the $p d e 6 c^{\text {w59 }}$ retina, native rods degenerate only in the central retina where the cone density is highest, suggesting that the density of dead or dying cones influences the death of nearby rods (Stearns et al., 2007). Comparing the viability of wild-type transplants in the central, intermediate and peripheral retina, we saw no difference in transplant viability as a function of location within the retina. Additionally, in the larval stages, rods are sparse within the central retina, generally occurring as isolated cells. We investigated whether the size of the transplant influenced the viability of wildtype cones. We found that smaller transplants had a slightly diminished viability, but this was independent of the genotype of the host, suggesting that it was a function of the transplantation rather than a bystander effect. Furthermore, in some cases, individual cones could be identified to have survived despite their isolation. Finally, we investigated the timing of bystander death 
A
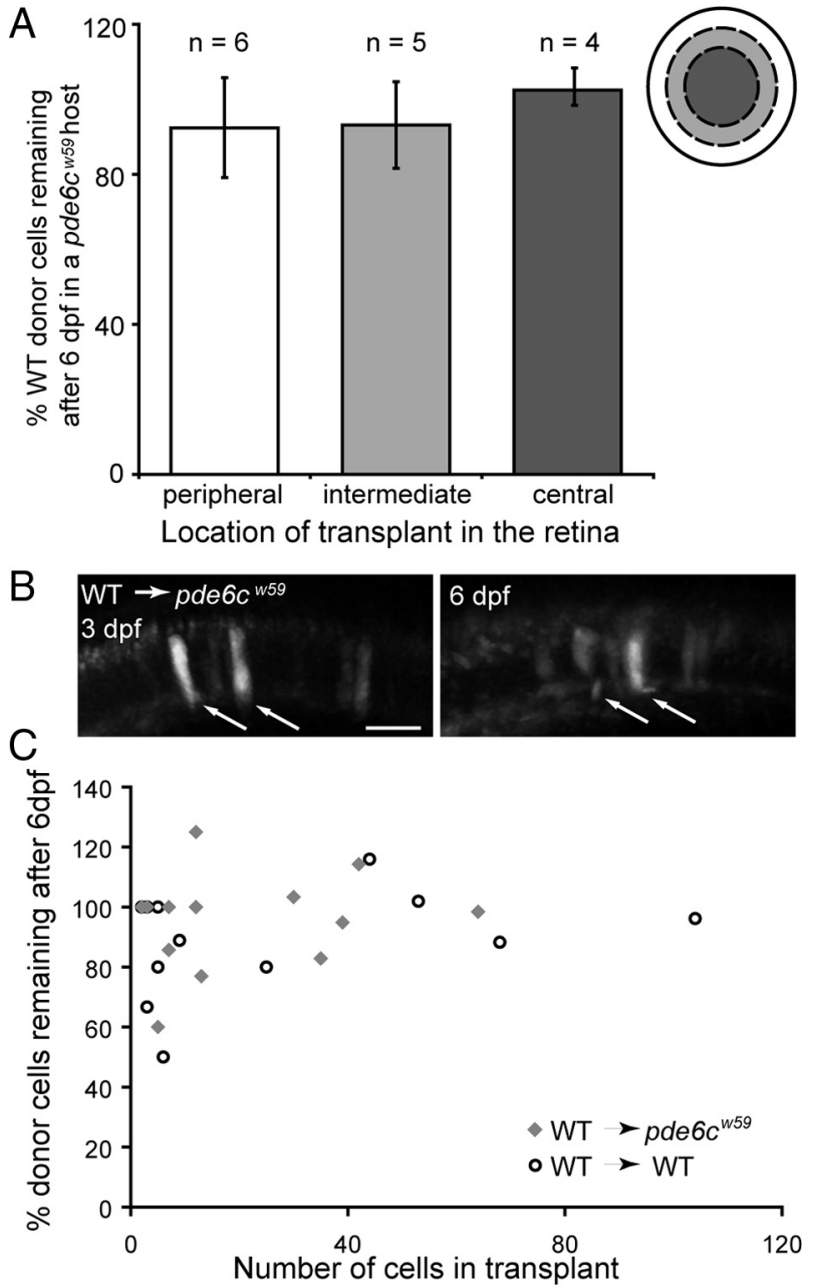

Figure 5. Location and size does not influence the viability of wild-type donors in the mutant hosts. A, Images from wild-type donors in $p d e 6 c^{\mathrm{w} 59}$ hosts were divided into the peripheral, intermediate, and central retina (see diagram). The viability of transplants in each region is shown as a percentage of cells from the $3 \mathrm{dpf}$ image that remained after $6 \mathrm{dpf}$. There was not a difference between regions. Error bars are the SEM, and $n=$ the number transplant patches. $\boldsymbol{B}, \boldsymbol{C}$, The size of the transplant does not influence wild-type viability. $\boldsymbol{B}$, Higher magnification images from $T g\left(T \alpha\right.$ CP:GFP) donors in $p d e 6 c^{\mathrm{w} 59} \mathrm{Tg}(\mathrm{T} \alpha(P: M C F P)$ host showing the continued presence of isolated individual donor cells at 3 and $6 \mathrm{dpf}$ (arrows). Scale bar, $10 \mu \mathrm{m}$. C, Threedimensional reconstructions of the image stacks were used to count the cones within isolated patches of wild-type transplant in either wild-type or $p d e 6 c^{\mathrm{w} 59}$ hosts. The viability of these transplants is graphed as a function of the number of cells in the patch at $3 \mathrm{dpf}$. No differences are seen between wild-type donors in the two host environments.

of neighboring cones and found that even after several weeks transplanted cones were viable in the mutant host. These data suggest that cones and rods are influenced differently by the death of neighboring cells. In particular it appears that the death of mutant cones does not affect WT cones even in regions where it causes the death of native rods. This implies that the signal governing the bystander effect in the retina is cell type-specific or is delivered in a cell-type-specific manner.

\section{Transmission of the bystander effect}

Multiple mechanisms have been proposed to explain the bystander effect within the retina. These can be separated into two general categories. First, the mutant population releases factors that influence the neighboring cells; specifically rods release a trophic factor that stimulates cone growth and maintenance. (Mohand-Said et al., 2000; Chalmel et al., 2007). Second, the

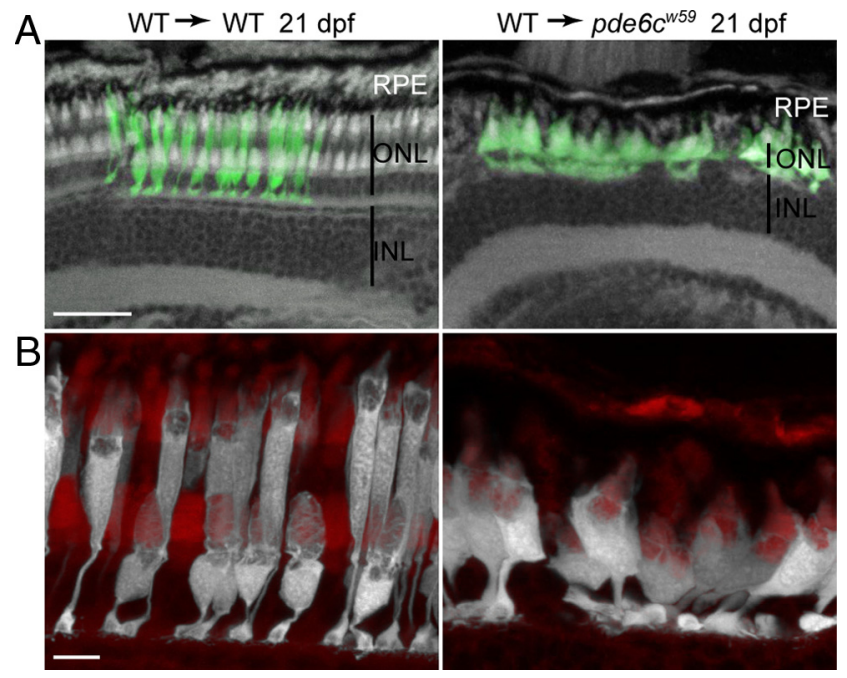

Figure 6. Transplanted wild-type cones are still present at $21 \mathrm{dpf}$. Chimeric fish were grown to $21 \mathrm{dpf}$, cryosectioned, and imaged. $A$, Low-magnification images show the retinal layers with the decreased size of the $\mathrm{ONL}$ in the mutant retina. Cones from wild-type donors expressing $T g(T \alpha$ (P:GFP) are shown in green. Scale bar, $50 \mu \mathrm{m}$. $\boldsymbol{B}$, High-magnification images show the morphology of the wild-type donor cones in gray scale. Wild-type cones in the mutant retina are shorter, presumably due to collapse of the ONL. Scale bar, $10 \mu \mathrm{m}$.

death of mutant cells results in environmental perturbation that kills other cells in the affected area. Potential sources of environmental perturbation include the activation of microglia involved in the removal of apoptotic bodies (Gupta et al., 2003), an increase in oxygen leading to reactive oxygen species (Komeima et al., 2007; Usui et al., 2009), and disruption of nutrient delivery resulting in the starvation of the remaining photoreceptors (Punzo et al., 2009). In all of these cases the density of dying cells strongly influences the levels of bystander death. We have seen a similar effect in our mutant with regard to rods in the central retina. However, our current data suggests that there are factors in addition to density that will affect the passage of death from one population to the next. The continued presence of wild-type cones in the central retina implies that, however the death of neighbors is mediated, it is either differentially targeted to the separate cell types, or it is a general effect to which rods and cones have a different response.

There are several possibilities for how cones and rods might be differentially targeted for bystander death. Evidence in other cell systems, and in the developing retina, have shown that gap junctions between cells are important for the bystander effect (Cusato et al., 2003; Krysko et al., 2005). Gap junctions provide a regulated flow of small molecules between cells that can be modified by the identity of the connexins that make up the pore. Different connexins have been shown to have different abilities to transmit a bystander effect (Andrade-Rozental et al., 2000). One possibility is that cone to cone gap junctions do not transmit the bystander signal while cone to rod and rod to rod junctions do. In this case the bystander signal could be a toxic factor, or it could be a trophic or nutritional factor. Another possibility is that cones and rods have different metabolic needs and thus respond differently to changes in the local environment. For example, retinal processing in rods is accomplished through the retinal pigmented epithelium (RPE), while processing in cones occurs both in the RPE and in Müller glia (Fleisch et al., 2008). Thus, rods might be more sensitive to perturbations of RPE attachment than cones.

The bystander effect in zebrafish has been evaluated in one other study (Morris et al., 2005). A transgene overexpressed in rods, $\operatorname{Tg}(X O P S: M C F P)$, led to the early death of rod photorecep- 
tors and subsequent neighboring cone death was evaluated. No cone death was detected in this transgenic rod mutant, even after many months. Although this finding might suggest fundamental differences in the transmission of bystander associated death in mammals and teleosts, simple differences in cell development and density more likely explain this result. Zebrafish are strongly cone dominated particularly at early ages, and rods develop more slowly, contributing significantly to visual responses only after 14 dpf (Branchek, 1984). In the Tg(XOPS:MCFP) strain, rods die rapidly after their formation starting at 3-4 dpf (Morris et al., 2005). At this age, zebrafish rods are sparse, found in isolated patches and scattered throughout the retina. As additional rods form they also rapidly die. Thus, the density of rods is always very low in this transgenic strain. This suggests that, similar to mammalian systems, a considerable density of dying cells may be required to stimulate a bystander response. Furthermore, the presence of rod death in the central retina of the $p d e 6 c^{\mathrm{w} 59}$ mutant following massive cone death in this region (Stearns et al., 2007) is consistent with a density requirement and indicates that bystander associated death can occur within the zebrafish retina.

\section{The bystander effect and transplantation}

The current therapeutic treatment for retinal degeneration is very limited; however, recently, important new advances have been developed in the transplantation of photoreceptors into the subretinal space. Using stem cells and retinal explants from early developmental stages, several labs have shown that transplanted cells will incorporate into existing retinal structures and even provide signaling to downstream cells (MacLaren et al., 2006; Peng et al., 2007). However, the levels of incorporation are very low: as few as $1 \%$ of the injected cells will migrate into the outer nuclear layer (MacLaren et al., 2006). Human and animal models indicate that even a very small number of functional photoreceptors can positively influence visual acuity. Thus, despite the low levels of integration, transplantation remains one of the most promising potential therapies for retinal degeneration.

The bystander effect in the retina represents a significant potential threat to this method of retinal replacement. Since only a small number of new retinal cells are incorporated into the damaged retina, it is important that these cells remain healthy and not be damaged by further degeneration of the neighboring cells. Our data suggests that wild-type cones are able to survive in an environment dominated by dying mutant cones regardless of the wild-type clone size. Thus, even isolated cones incorporated into the fovea may not be sensitive to the death of other cones. Many challenges still remain in the implementation of human transplants, but our data suggests one less hurdle to be overcome before this therapy is a reality.

\section{References}

Andrade-Rozental AF, Rozental R, Hopperstad MG, Wu JK, Vrionis FD, Spray DC (2000) Gap junctions: the "kiss of death" and the "kiss of life." Brain Res Brain Res Rev 32:308-315.

Branchek T (1984) The development of photoreceptors in the zebrafish, brachydanio rerio. II. Function. J Comp Neurol 224:116-122.

Brockerhoff SE (2006) Measuring the optokinetic response of zebrafish larvae. Nat Protoc 1:2448-2451.

Carmany-Rampey A, Moens CB (2006) Modern mosaic analysis in the zebrafish. Methods 39:228-238.

Chalmel F, Léveillard T, Jaillard C, Lardenois A, Berdugo N, Morel E, Koehl P, Lambrou G, Holmgren A, Sahel JA, Poch O (2007) Rod-derived Cone Viability Factor-2 is a novel bifunctional-thioredoxin-like protein with therapeutic potential. BMC Mol Biol 8:74.

Cusato K, Bosco A, Rozental R, Guimarães CA, Reese BE, Linden R, Spray DC (2003) Gap junctions mediate bystander cell death in developing retina. J Neurosci 23:6413-6422.
Delyfer MN, Léveillard T, Mohand-Saïd S, Hicks D, Picaud S, Sahel JA (2004) Inherited retinal degenerations: therapeutic prospects. Biol Cell 96:261-269.

Fleisch VC, Schonthaler HB, von Lintig J, Neuhauss SC (2008) Subfunctionalization of a retinoid-binding protein provides evidence for two parallel visual cycles in the cone-dominant zebrafish retina. J Neurosci 28:8208-8216.

Gupta N, Brown KE, Milam AH (2003) Activated microglia in human retinitis pigmentosa, late-onset retinal degeneration, and age-related macular degeneration. Exp Eye Res 76:463-471.

Hamel CP (2007) Cone rod dystrophies. Orphanet J Rare Dis 2:7.

Hartong DT, Berson EL, Dryja TP (2006) Retinitis pigmentosa. Lancet 368:1795-1809.

Huang PC, Gaitan AE, Hao Y, Petters RM, Wong F (1993) Cellular interactions implicated in the mechanism of photoreceptor degeneration in transgenic mice expressing a mutant rhodopsin gene. Proc Natl Acad Sci U S A 90:8484-8488.

Kedzierski W, Bok D, Travis GH (1998) Non-cell-autonomous photoreceptor degeneration in rds mutant mice mosaic for expression of a rescue transgene. J Neurosci 18:4076-4082.

Kennedy BN, Alvarez Y, Brockerhoff SE, Stearns GW, Sapetto-Rebow B, Taylor MR, Hurley JB (2007) Identification of a zebrafish cone photoreceptor-specific promoter and genetic rescue of achromatopsia in the nof mutant. Invest Ophthalmol Vis Sci 48:522-529.

Komeima K, Rogers BS, Campochiaro PA (2007) Antioxidants slow photoreceptor cell death in mouse models of retinitis pigmentosa. J Cell Physiol 213:809-815

Krysko DV, Leybaert L, Vandenabeele P, D’Herde K (2005) Gap junctions and the propagation of cell survival and cell death signals. Apoptosis 10:459-469.

Kwan KM, Fujimoto E, Grabher C, Mangum BD, Hardy ME, Campbell DS, Parant JM, Yost HJ, Kanki JP, Chien CB (2007) The Tol2kit: a multisite gateway-based construction kit for Tol2 transposon transgenesis constructs. Dev Dyn 236:3088-3099.

MacLaren RE, Pearson RA, MacNeil A, Douglas RH, Salt TE, Akimoto M, Swaroop A, Sowden JC, Ali RR (2006) Retinal repair by transplantation of photoreceptor precursors. Nature 444:203-207.

Michaelides M, Hunt DM, Moore AT (2004) The cone dysfunction syndromes. Br J Ophthalmol 88:291-297.

Mohand-Said S, Hicks D, Dreyfus H, Sahel JA (2000) Selective transplantation of rods delays cone loss in a retinitis pigmentosa model. Arch Ophthalmol 118:807-811.

Morris AC, Schroeter EH, Bilotta J, Wong RO, Fadool JM (2005) Cone survival despite rod degeneration in XOPS-mCFP transgenic zebrafish. Invest Ophthalmol Vis Sci 46:4762-4771.

Peng Q, Thomas BB, Aramant RB, Chen Z, Sadda SR, Seiler MJ (2007) Structure and function of embryonic rat retinal sheet transplants. Curr Eye Res 32:781-789.

Punzo C, Kornacker K, Cepko CL (2009) Stimulation of the insulin/mTOR pathway delays cone death in a mouse model of retinitis pigmentosa. Nat Neurosci 12:44-52.

Ren JQ, McCarthy WR, Zhang H, Adolph AR, Li L (2002) Behavioral visual responses of wild-type and hypopigmented zebrafish. Vision Res 42:293-299.

Ripps H (2002) Cell death in retinitis pigmentosa: gap junctions and the 'bystander' effect. Exp Eye Res 74:327-336.

Stearns G, Evangelista M, Fadool JM, Brockerhoff SE (2007) A mutation in the cone specific pde6 gene causes rapid cone photoreceptor degeneration in zebrafish. J Neurosci 27:13866-13874.

Taylor MR, Kikkawa S, Diez-Juan A, Ramamurthy V, Kawakami K, Carmeliet P, Brockerhoff SE (2005) The zebrafish pob gene encodes a novel protein required for survival of red cone photoreceptor cells. Genetics 170:263-273.

Truett GE, Heeger P, Mynatt RL, Truett AA, Walker JA, Warman ML (2000) Preparation of PCR-quality mouse genomic DNA with hot sodium hydroxide and tris (HotSHOT). Biotechniques 29:52, 54.

Usui S, Oveson BC, Lee SY, Jo YJ, Yoshida T, Miki A, Miki K, Iwase T, Lu L, Campochiaro PA (2009) NADPH oxidase plays a central role in cone cell death in retinitis pigmentosa. J Neurochem 110:1028-1037.

Westerfield M (1995) The zebrafish book: a guide for the laboratory use of zebrafish (Brachydanio rerio). Eugene, OR: University of Oregon. 